\title{
Effect of Supplementation of Tinospora cordifolia and Ascorbic Acid Combinations on Growth Performance of Broiler Chickens
}

\author{
Dinesh Jain \\ Department of Animal Nutrition, College of Veterinary and Animal Science, Bikaner, Rajasthan, INDIA \\ Corresponding author: D Jain; E-mail: ddineshvet@gmail.com
}

Received: 04 Sept., 2021

Revised: 23 Sept., 2021

Accepted: 27 Sept., 2021

\begin{abstract}
The present study was carried out to investigate the effect of supplementation of graded levels of Tinospora cordifolia with or without ascorbic acid on the growth performance of broilers. A total of 360 one-d-old broiler chicks were assigned randomly to ten dietary groups $\left(\mathrm{T}_{1}-\mathrm{T}_{10}\right)$ in a $5 \times 2$ factorial arrangement, with three replicates of 12 chicks. The 42 -day feeding trial involved feeding of a basal diet supplemented with graded levels of Tinospora cordifolia with or without ascorbic acid. The average ambient temperature during the study period was $31^{\circ} \mathrm{C}$. Chronic heat stress in present study showed significant negative effect on feed conversion ratio (FCR) in control group as compared to various treatment groups. Significant effect of Tinospora cordifolia or ascorbic acid alone and their various combinations was observed on overall feed intake, body weight gain and feed conversion ratio (FCR). It was concluded that supplementation of Tinospora cordifolia at $0.25 \%$ level with ascorbic acid @ $0.025 \%$ is quite effective and could be viable proposition for lucrative broiler farming for meat production.
\end{abstract}

\section{HIGHLIGHTS}

( Supplementation of Tinospora cordifolia at 0.25\% level with ascorbic acid @ $0.025 \%$ is quite effective.

0 It could be viable proposition for lucrative broiler farming for meat production.

Keywords: Ascorbic acid, Growth performance, Heat stress, Tinospora cordifolia

The use of natural products has become increasingly popular in human as well as in livestock due to their multiple positive effects on the health (Puvaca et al., 2013). Tinospora cordifolia is a well-known traditional medicinal plant in India, which belongs to the family of Menispermaceae. The herb is known for its growth promoting, general health improvement of the birds, powerful therapeutic and prophylactic effects (Guilhelmelli et al., 2013 and Saeed et al., 2018). Another feed additive, vitamin $\mathrm{C}$, is an effective scavenger of reactive oxygen species, ascorbic acid minimizes the oxidative stress associated with the activated phagocytic leukocytes, thus checking the inflammation and tissue damage associated with immune responses. Adequate and appropriate nutritional strategy is required for optimal broiler production in the hot season, which can reduce the negative effects of heat stress to the minimum. Therefore, the present study was planned to investigate the effect of supplementation of graded levels of Tinospora cordifolia alone or in combination with ascorbic acid on the growth performance of broiler chickens during hot climate.

\section{MATERIALS AND METHODS}

A 42 days feeding trial was carried out at the Experimental Poultry Unit, located in poultry farm of College of Veterinary and Animal Science, Bikaner. The 360 experimental day old broiler chicks were equally and randomly divided into ten dietary treatments groups

How to cite this article: Jain, D. (2021). Effect of Supplementation of Tinospora cordifolia and Ascorbic Acid Combinations on Growth Performance of Broiler Chickens. J. Anim. Res., 11(05): 925-932.

Source of Support: None; Conflict of Interest: None $\rightarrow$ 
$\left(\mathrm{T}_{1}-\mathrm{T}_{10}\right)$ and each dietary group was replicated to three sub-groups $\left(R_{1}-R_{3}\right)$ to make sure uniformly in various treatment groups. Diets included: $\mathrm{T}_{1}$-basal diet with no supplementation; $\mathrm{T}_{2}$-basal diet supplemented with $0.25 \%$ Tinospora cordifolia; $\mathrm{T}_{3}$-basal diet supplemented with $0.50 \%$ Tinospora cordifolia; $\mathrm{T}_{4}$-basal diet supplemented with $0.75 \%$ Tinospora cordifolia; $\mathrm{T}_{5}$-basal diet supplemented with $1.0 \%$ Tinospora cordifolia; $\mathrm{T}_{6}$-basal diet supplemented with $0.025 \%$ ascorbic acid; $\mathrm{T}_{7}$-basal diet supplemented with $0.25 \%$ Tinospora cordifolia plus $0.025 \%$ ascorbic acid; $\mathrm{T}_{8}$-basal diet supplemented with $0.50 \%$ Tinospora cordifolia plus $0.025 \%$ ascorbic acid; $\mathrm{T}_{9}$-basal diet supplemented with $0.75 \%$ Tinospora cordifolia plus $0.025 \%$ ascorbic acid and $\mathrm{T}_{10}$-basal diet supplemented with $1.0 \%$ Tinospora cordifolia plus $0.025 \%$ ascorbic acid. Broilers were maintained under standard managemental practices and disease control throughout the trial period. The commercially available ascorbic acid (99.99\% pure), was used. Good quality of Tinospora cordifolia stem was procured from reputed firm of Bikaner (Rajasthan). The GC-MS full scan analysis of chloroform extracts of Tinospora cordifolia stem was carried out as analytical column-TG-5MS (Thermo-Scientific). The spectra were recorded for mass range $50-550 \mathrm{~m} / \mathrm{z}$ for about 50 minutes. The proximate analysis of experimental feed mixtures was carried out by the methods of AOAC (2005) and the minerals i.e. calcium and phosphorous were estimated by ICP-OES. During the study period, digital thermohygrometer was used to record temperature and relative humidity thrice a day at 08:00, 14:00 and 22:00 hours, respectively. THI values were calculated from the recorded measurement as described below by (Kibler, 1964). The chicks were weighed individually at the start of the experiment and subsequently at weekly intervals for 6 weeks. Feed consumption and body weight gain was recorded at weekly intervals for 6 weeks and feed conversion ratio (FCR) was calculated by dividing the cumulative feed intake by body weight gain of chicks for every week.

\section{Statistical analysis}

The data obtained in the experiment were analysed statistically for main effect of Tinospora cordifolia or ascorbic acid alone as well as interaction (Tinospora cordifolia $\times$ Ascorbic acid) in factorial design $\left(5^{*} 2\right)$ as per Snedecor and Cochran (2004) and significance of mean differences was tested by Duncan's New Multiple Range Test (DNMRT) as modified by Kramer (1957).

\section{RESULTS AND DISCUSSION}

Major phyto-components identified in chloroform extract of Tinospora cordifolia with their chemical structure, nature of compounds and medicinal uses have been presented in Table 1. The mean values of temperature $\left({ }^{\circ} \mathrm{C}\right)$, relative humidity $(\%)$ and temperature humidity index (THI) recorded during different weeks have been presented in Table 2. The calculated THI for different weeks was obtained to be in range from 75.26 to 82.06 ; a value above the THI threshold of 70, established for poultry (Karaman et al., 2007). The average temperature $\left(31^{\circ} \mathrm{C}\right)$ during the study period was higher than the recommended normothermia zone i.e. $22-28^{\circ} \mathrm{C}$ (Donkoh, 1989) established for poultry in the tropical regions, which indicated that birds were in chronic heat stress. The chemical composition of different experimental feed offered in starter and finisher phase to the broilers have been presented in Table 3. The proximate composition of different experimental feeds was almost similar for all the treatment groups and fed as per BIS (1992). The data of weekly feed intake, body weight gain and feed conversion efficiency of broilers observed in various treatment groups have been presented in Table 4, 5 and 6 respectively. The statistical analysis of data of feed intake due to main effect of Tinospora cordifolia and ascorbic acid as well as interaction revealed highly significant $(\mathrm{P}<0.01)$ effect. The feed intake recorded for entire period of six weeks was highest for $\mathrm{T}_{8}$ group (3951.19 g) which was statistically comparable with $\mathrm{T}_{9}$, and $\mathrm{T}_{10}$, but significantly higher than rest of the groups. The statistical analysis of data of overall body weight gain due to main effect of Tinospora cordifolia and ascorbic acid as well as interaction revealed highly significant $(\mathrm{P}<0.01)$ effect. The highest mean body weight gain recorded for entire period of experiment i.e. for VI weeks in $\mathrm{T}_{7}(2372.67 \mathrm{~g})$ containing $0.25 \%$ Tinospora cordifolia with ascorbic acid, which was though statistically comparable with $\mathrm{T}_{8}$, but significantly higher $(\mathrm{P}<0.01)$ than rest of the groups. The statistical analysis of data of FCR due to main effect of Tinospora cordifolia and ascorbic acid as well as 
Table 1: Activity of phyto-components identified in chloroform extract of Tinospora cordifolia

\begin{tabular}{|c|c|c|c|c|}
\hline \multirow{2}{*}{ Sl. No. } & \multirow{2}{*}{ Compound Name } & \multicolumn{3}{|c|}{ Chloroform extract of Tinospora cordifolia } \\
\hline & & Structure & Nature & Uses \\
\hline & $\begin{array}{l}\text { Vanillin lactoside (4-Formyl- } \\
\text { 2-methoxyphenyl } \\
\text { 4-O-hexopyranosylhexopyranoside) }\end{array}$ & & Phenolic aldehyde & $\begin{array}{l}\text { Flavoring agent, Antioxidant } \\
\text { activity, Antimicrobial } \\
\text { Antidiabetic activity, Anti } \\
\text { inflammatory activity, } \\
\text { Analgesic activity, Anticancer }\end{array}$ \\
\hline & $\begin{array}{l}\text { Hexadecanoic acid, methyl ester (Palmitic } \\
\text { acid, methyl ester) }\end{array}$ & $M$ & Fatty acid & $\begin{array}{l}\text { Flavoring Agents, Antioxidents, } \\
\text { Surface active agents }\end{array}$ \\
\hline & 1-(+)-Ascorbic acid 2, 6 dihexadecanoate & & $\begin{array}{l}\text { Sugar acid } \\
\text { (Vitamin } \\
\text { derivative) }\end{array}$ & Antioxidant \\
\hline & Octadecanoic acid & & Stearic acid & $\begin{array}{l}\text { Antiviral and Anti- } \\
\text { inflammatory activities, } \\
\text { Flavoring Agents, } \\
\text { Hypocholesterolemic }\end{array}$ \\
\hline & $\begin{array}{l}\text { Columbin(1,4-Etheno-3H,7H-benzo[1, } \\
\text { 2-c:3, 4-c']dipyran-3, 7-dione, } \\
\text { 9-(3-furanyl) decahydro-4-hydroxy- } \\
\text { 4a,10a-dimethyl-,[1R(1à, 4á, 4aà, 6aá, 9á, } \\
\text { 10aá, 10bà)]-) }\end{array}$ & & $\begin{array}{l}\text { Diterpenoid } \\
\text { lactone }\end{array}$ & $\begin{array}{l}\text { Antimicrobial and } \\
\text { Antiinflammatory }\end{array}$ \\
\hline & Campesterol & & Phytosterol & $\begin{array}{l}\text { Antimicrobial, Antioxidants, } \\
\text { Hypocholesterolemic, } \\
\text { Antiatheroscleroic, Antiasthma, } \\
\text { Antiartritic }\end{array}$ \\
\hline & $\begin{array}{l}\text { Stigmasterol (Stigmasta-5, 22-dien-3-ol, } \\
(3 a ́, 22 \mathrm{E})-)\end{array}$ & & Phytosterol & $\begin{array}{l}\text { Antimicrobial and antioxidants, } \\
\text { cholesterol-lowering properties. }\end{array}$ \\
\hline & $\begin{array}{l}\text { ç-Sitosterol (Stigmast-5-en-3-ol, (3á, } \\
\text { 24S)-) }\end{array}$ & & Steroid & $\begin{array}{l}\text { Antimicrobial, Anticancer, } \\
\text { Antiarthritic, Antiasthma, } \\
\text { Diuretic and Antiinflammatory }\end{array}$ \\
\hline & á-Amyrin (Olean-12-en-3-ol, (3á)-) & & Triterpine & $\begin{array}{l}\text { Hepatoprotective, Prevent } \\
\text { oxidative stress, Apoptosis, } \\
\text { Anti-inflammatory and Anti- } \\
\text { fibrogenic }\end{array}$ \\
\hline
\end{tabular}


Table 2: Mean temperature $\left({ }^{\circ} \mathrm{C}\right)$, relative humidity $(\%)$ and THI range observed during different weeks

\begin{tabular}{|c|c|c|c|c|c|c|c|c|c|c|c|c|}
\hline \multirow{2}{*}{$\begin{array}{l}\text { Period } \\
\text { (weeks) }\end{array}$} & \multicolumn{4}{|c|}{ Temperature $\left({ }^{\circ} \mathrm{C}\right)$} & \multicolumn{4}{|c|}{ Relative humidity (\%) } & \multicolumn{4}{|l|}{ THI } \\
\hline & 8 am & $2 \mathrm{pm}$ & $10 \mathrm{pm}$ & Avg. & 8 am & $2 \mathrm{pm}$ & $10 \mathrm{pm}$ & Avg. & 8 am & 2 pm & $10 \mathrm{pm}$ & Avg. \\
\hline I & 32.24 & 35.54 & 31.44 & 33.08 & 53.30 & 42.09 & 53.21 & 49.53 & 81.66 & 83.68 & 80.58 & 82.06 \\
\hline II & 31.13 & 33.40 & 33.83 & 32.79 & 50.86 & 42.71 & 39.00 & 44.19 & 79.76 & 81.18 & 80.98 & 80.70 \\
\hline III & 30.59 & 32.87 & 32.84 & 32.10 & 58.43 & 52.71 & 44.71 & 51.95 & 80.28 & 82.39 & 80.87 & 81.23 \\
\hline IV & 28.94 & 31.14 & 29.87 & 29.99 & 66.71 & 59.00 & 60.14 & 61.95 & 79.22 & 81.15 & 79.56 & 80.01 \\
\hline V & 27.16 & 30.46 & 29.53 & 29.05 & 46.43 & 43.29 & 42.00 & 43.90 & 74.00 & 77.66 & 76.32 & 76.01 \\
\hline VI & 27.45 & 33.31 & 26.40 & 29.05 & 46.03 & 27.30 & 42.94 & 38.76 & 74.31 & 78.13 & 72.62 & 75.26 \\
\hline Mean & 29.58 & 32.79 & 30.65 & 31.00 & 53.63 & 44.52 & 47.00 & 48.38 & 78.21 & 80.70 & 78.49 & 79.21 \\
\hline
\end{tabular}

Table 3: The chemical (\% DM basis) composition of experimental feed offered during feeding trial to broiler chicks

\begin{tabular}{|c|c|c|c|c|c|c|c|c|c|c|}
\hline \multirow{2}{*}{ Parameters } & \multicolumn{10}{|c|}{ Treatment groups } \\
\hline & $\mathbf{T}_{1}$ & $\mathbf{T}_{2}$ & $\mathbf{T}_{3}$ & $\mathbf{T}_{4}$ & $T_{5}$ & $\mathrm{~T}_{6}$ & $\mathbf{T}_{7}$ & $\mathbf{T}_{8}$ & $T_{9}$ & $T_{10}$ \\
\hline \multicolumn{11}{|c|}{ Broiler starter } \\
\hline $\mathrm{DM}$ & 91.15 & 91.16 & 91.16 & 91.17 & 91.17 & 91.13 & 91.13 & 91.14 & 91.14 & 91.15 \\
\hline $\mathrm{CP}$ & 21.37 & 21.32 & 21.27 & 21.22 & 21.17 & 21.36 & 21.31 & 21.26 & 21.21 & 21.16 \\
\hline $\mathrm{EE}$ & 4.04 & 4.03 & 4.02 & 4.02 & 4.01 & 4.04 & 4.03 & 4.02 & 4.02 & 4.01 \\
\hline $\mathrm{CF}$ & 3.75 & 3.77 & 3.80 & 3.83 & 3.85 & 3.75 & 3.77 & 3.80 & 3.83 & 3.85 \\
\hline TA & 6.70 & 6.70 & 6.71 & 6.71 & 6.71 & 6.70 & 6.70 & 6.70 & 6.71 & 6.71 \\
\hline AIA & 1.25 & 1.25 & 1.25 & 1.25 & 1.25 & 1.25 & 1.25 & 1.25 & 1.25 & 1.25 \\
\hline NDF & 9.98 & 10.01 & 10.05 & 10.08 & 10.12 & 9.98 & 10.01 & 10.05 & 10.08 & 10.11 \\
\hline $\mathrm{ADF}$ & 3.25 & 3.30 & 3.35 & 3.41 & 3.46 & 3.25 & 3.30 & 3.35 & 3.41 & 3.46 \\
\hline $\mathrm{Ca}$ & 1.14 & 1.14 & 1.14 & 1.13 & 1.13 & 1.14 & 1.14 & 1.14 & 1.13 & 1.13 \\
\hline $\mathrm{P}$ & 1.18 & 1.18 & 1.18 & 1.17 & 1.17 & 1.18 & 1.18 & 1.17 & 1.17 & 1.17 \\
\hline \multicolumn{11}{|c|}{ Broiler finisher } \\
\hline $\mathrm{DM}$ & 91.41 & 91.42 & 91.42 & 91.43 & 91.43 & 91.39 & 91.39 & 91.40 & 91.40 & 91.41 \\
\hline $\mathrm{CP}$ & 20.32 & 20.27 & 20.23 & 20.18 & 20.13 & 20.32 & 20.27 & 20.22 & 20.17 & 20.13 \\
\hline $\mathrm{EE}$ & 4.60 & 4.59 & 4.58 & 4.58 & 4.57 & 4.60 & 4.59 & 4.58 & 4.57 & 4.57 \\
\hline $\mathrm{CF}$ & 3.75 & 3.78 & 3.80 & 3.83 & 3.85 & 3.75 & 3.78 & 3.80 & 3.83 & 3.85 \\
\hline TA & 6.41 & 6.41 & 6.41 & 6.41 & 6.42 & 6.41 & 6.41 & 6.41 & 6.41 & 6.41 \\
\hline AIA & 1.35 & 1.35 & 1.35 & 1.35 & 1.34 & 1.35 & 1.35 & 1.35 & 1.35 & 1.34 \\
\hline NDF & 10.21 & 10.24 & 10.28 & 10.31 & 10.34 & 10.21 & 10.24 & 10.27 & 10.31 & 10.34 \\
\hline $\mathrm{ADF}$ & 3.43 & 3.48 & 3.53 & 3.59 & 3.64 & 3.43 & 3.48 & 3.53 & 3.58 & 3.64 \\
\hline $\mathrm{Ca}$ & 0.86 & 0.86 & 0.86 & 0.86 & 0.85 & 0.86 & 0.86 & 0.86 & 0.85 & 0.85 \\
\hline $\mathrm{P}$ & 0.91 & 0.91 & 0.91 & 0.90 & 0.90 & 0.91 & 0.91 & 0.91 & 0.90 & 0.90 \\
\hline
\end{tabular}

interaction revealed highly significant $(\mathrm{P}<0.01)$ effect. The FCR for entire experimental period i.e. VI weeks was recorded significantly $(\mathrm{P}<0.01)$ lowest in $\mathrm{T}_{7}$ containing $0.25 \%$ Tinospora cordifolia with ascorbic acid.

The results obtained in present study regarding feed intake are in accordance with findings of Singh et al. (2009), Kulkarni et al. (2011) and Bhushan et al. (2013) who reported significant increase in feed intake when broilers were supplemented with Tinospora cordifolia stem powder in ration. The increased feed intake in Tinospora cordifolia supplemented groups might be due 
Table 4: Effect of supplementation of Tinospora cordifolia and ascorbic acid on feed intake (g) at different weeks

\begin{tabular}{|c|c|c|c|c|c|c|c|}
\hline \multirow{2}{*}{$\begin{array}{l}\text { Treatment } \\
\text { groups }\end{array}$} & \multicolumn{7}{|c|}{ Period (weeks) } \\
\hline & 0-I & I-II & II-III & III-IV & IV-V & V-VI & I-VI \\
\hline \multicolumn{8}{|c|}{ Tinospora cordifolia X Ascorbic acid } \\
\hline $\mathrm{T}_{1}$ & $160.56^{\mathrm{e}}$ & $318.89^{c}$ & $579.39^{\mathrm{a}}$ & $685.83^{\mathrm{a}}$ & 852.39 & $968.97^{\mathrm{b}}$ & $3566.03^{\mathrm{a}}$ \\
\hline $\mathrm{T}_{2}$ & $154.50^{\mathrm{abc}}$ & $311.389^{\mathrm{bc}}$ & $575.03^{\mathrm{a}}$ & $789.42^{\mathrm{f}}$ & 870.67 & $1043.22^{\mathrm{e}}$ & $3744.22^{c}$ \\
\hline $\mathrm{T}_{3}^{2}$ & $158.56^{\mathrm{de}}$ & $306.53^{\mathrm{ab}}$ & $569.92^{\mathrm{a}}$ & $743.08^{\mathrm{bc}}$ & 858.92 & $1019.81^{d}$ & $3656.81^{b}$ \\
\hline $\mathrm{T}_{4}$ & $167.50^{\mathrm{f}}$ & $298.58^{\mathrm{a}}$ & $571.44^{\mathrm{a}}$ & $743.19^{b}$ & 831.58 & $985.72^{\mathrm{bc}}$ & $3598.03^{\mathrm{ab}}$ \\
\hline $\mathrm{T}_{5}$ & $165.94^{\mathrm{g}}$ & $297.78^{\mathrm{a}}$ & $570.78^{a}$ & $742.78^{b c}$ & 830.97 & $984.86^{\mathrm{bc}}$ & $3593.11^{\mathrm{ab}}$ \\
\hline $\mathrm{T}_{6}$ & $157.19^{\mathrm{bcd}}$ & $306.89^{a b}$ & $617.61^{b}$ & $739.53^{b}$ & 879.31 & $884.75^{\mathrm{a}}$ & $3585.28^{\mathrm{a}}$ \\
\hline $\mathrm{T}_{7}^{0}$ & $153.47^{\mathrm{ab}}$ & $360.28^{\mathrm{e}}$ & $686.33^{\text {ce }}$ & $776.17^{\text {def }}$ & 940.78 & $989.17^{\mathrm{c}}$ & $3906.19^{d}$ \\
\hline $\mathrm{T}_{8}$ & $158.33^{\mathrm{cd}}$ & $367.56^{\mathrm{e}}$ & $692.14^{\mathrm{d}}$ & $761.94^{\mathrm{ce}}$ & 952.72 & $1018.50^{\mathrm{d}}$ & $3951.19^{f}$ \\
\hline $\mathrm{T}_{9}$ & $156.11^{\mathrm{bcd}}$ & $346.28^{d}$ & $699.08^{\mathrm{de}}$ & $765.58^{d}$ & 926.08 & $1057.28^{\mathrm{ef}}$ & $3950.42^{\mathrm{ef}}$ \\
\hline $\mathrm{T}_{10}^{9}$ & $151.61^{\mathrm{a}}$ & $310.19^{\mathrm{bc}}$ & $731.25^{f}$ & $734.75^{b}$ & 942.00 & $1061.61^{\mathrm{f}}$ & $3931.42^{\mathrm{ef}}$ \\
\hline SEM & 0.3599 & 1.0139 & 1.9963 & 1.7910 & 4.7539 & 1.6558 & 6.2058 \\
\hline \multicolumn{8}{|c|}{ Effect of Tinospora cordifolia } \\
\hline $0 \%$ & $158.88^{\mathrm{b}}$ & $312.89^{b}$ & $598.50^{\mathrm{a}}$ & $712.68^{\mathrm{a}}$ & 865.85 & $926.86^{\mathrm{a}}$ & $3575.66^{\mathrm{a}}$ \\
\hline $0.25 \%$ & $153.99^{\mathrm{a}}$ & $335.83^{d}$ & $630.68^{b}$ & $782.79^{d}$ & 905.72 & $1016.19^{b}$ & $3825.21^{\mathrm{c}}$ \\
\hline $0.50 \%$ & $158.44^{\mathrm{b}}$ & $337.04^{\mathrm{d}}$ & $631.03^{b}$ & $752.51^{\mathrm{c}}$ & 905.82 & $1019.15^{b}$ & $3804.00^{\mathrm{bc}}$ \\
\hline $0.75 \%$ & $161.81^{\mathrm{c}}$ & $322.43^{c}$ & $635.26^{\mathrm{b}}$ & $754.39^{c}$ & 878.83 & $1021.50^{\mathrm{b}}$ & $3774.22^{b}$ \\
\hline $1 \%$ & $158.78^{b}$ & $303.99^{\mathrm{a}}$ & $651.01^{\mathrm{c}}$ & $738.76^{\mathrm{b}}$ & 886.49 & $1023.24^{b}$ & $3762.26^{\mathrm{b}}$ \\
\hline SEM & 0.8816 & 2.4834 & 4.8800 & 4.3871 & 11.6445 & 4.0558 & 15.2011 \\
\hline \multicolumn{8}{|c|}{ Effect of Ascorbic acid } \\
\hline $0 \%$ & $161.41^{b}$ & $306.63^{\mathrm{a}}$ & $573.31^{\mathrm{a}}$ & $740.86^{\mathrm{a}}$ & $848.91^{\mathrm{a}}$ & 1000.52 & $3631.64^{\mathrm{a}}$ \\
\hline $0.025 \%$ & $155.34^{\mathrm{a}}$ & $338.24^{\mathrm{b}}$ & $685.28^{\mathrm{b}}$ & $755.59^{b}$ & $928.18^{\mathrm{b}}$ & 1002.26 & $3864.90^{\mathrm{b}}$ \\
\hline SEM & 0.5575 & 1.5706 & 3.0927 & 2.7747 & 7.3647 & 2.5651 & 9.6140 \\
\hline
\end{tabular}

Table 5: Effect of supplementation of Tinospora cordifolia and ascorbic acid on body weight gain (g) at different weeks

\begin{tabular}{|c|c|c|c|c|c|c|c|c|}
\hline \multirow{2}{*}{$\begin{array}{l}\text { Treatment } \\
\text { groups }\end{array}$} & \multicolumn{4}{|c|}{ Period (weeks) } & \multirow[b]{2}{*}{ III-IV } & \multirow[b]{2}{*}{ IV-V } & \multirow[b]{2}{*}{ V-VI } & \multirow[b]{2}{*}{ I-VI } \\
\hline & $\begin{array}{l}\text { Initial body } \\
\text { weight }\end{array}$ & 0-I & I-II & II-III & & & & \\
\hline \multicolumn{9}{|c|}{ Tinospora cordifolia X Ascorbic acid } \\
\hline $\mathrm{T}_{1}$ & & 117.56 & $192.08^{\mathrm{a}}$ & $322.03^{\mathrm{ab}}$ & $383.72^{\mathrm{a}}$ & $408.08^{\mathrm{a}}$ & $438.17^{\mathrm{a}}$ & $1861.64^{\mathrm{a}}$ \\
\hline $\mathrm{T}_{2}$ & & 125.72 & $210.61^{\mathrm{c}}$ & $327.44^{b}$ & $444.69^{c d}$ & $474.28^{c}$ & $485.28^{\mathrm{d}}$ & $2068.03^{\mathrm{d}}$ \\
\hline $\mathrm{T}_{3}$ & & 124.17 & $201.56^{b}$ & $320.94^{\mathrm{ab}}$ & $420.92^{b}$ & $464.17^{\mathrm{c}}$ & $472.47^{\mathrm{c}}$ & $2004.22^{c}$ \\
\hline $\mathrm{T}_{4}$ & & 128.94 & $196.03^{\mathrm{ab}}$ & $317.64^{\mathrm{a}}$ & $421.97^{b}$ & $438.00^{\mathrm{b}}$ & $452.06^{\mathrm{b}}$ & $1954.64^{\mathrm{b}}$ \\
\hline $\mathrm{T}_{5}$ & & 122.36 & $193.14^{\mathrm{ab}}$ & $315.61^{\mathrm{a}}$ & $423.47^{\mathrm{b}}$ & $447.17^{\mathrm{b}}$ & $438.14^{\mathrm{a}}$ & $1939.89^{b}$ \\
\hline $\mathrm{T}_{6}$ & & 119.97 & $210.19^{c}$ & $391.22^{\mathrm{c}}$ & $431.83^{b}$ & $506.56^{\mathrm{d}}$ & $442.22^{\mathrm{ab}}$ & $2102.00^{\mathrm{e}}$ \\
\hline $\mathrm{T}_{7}$ & & 122.86 & $268.89^{f}$ & $439.50^{\mathrm{ef}}$ & $470.25^{\mathrm{f}}$ & $554.53^{f g}$ & $516.64^{\mathrm{g}}$ & $2372.67^{\mathrm{h}}$ \\
\hline $\mathrm{T}_{8}$ & & 126.92 & $262.42^{\mathrm{ef}}$ & $437.78^{\mathrm{ef}}$ & $456.47^{\text {de }}$ & $553.03^{\mathrm{ef}}$ & $515.67^{f}$ & $2352.28^{\mathrm{gh}}$ \\
\hline $\mathrm{T}_{9}^{\circ}$ & & 126.58 & $257.69^{e}$ & $434.67^{d}$ & $449.55^{\mathrm{d}}$ & $556.22^{\mathrm{fg}}$ & $514.00^{\mathrm{fg}}$ & $2338.72^{\mathrm{g}}$ \\
\hline $\mathrm{T}_{10}$ & & 122.53 & $231.06^{\mathrm{d}}$ & $443.50^{\mathrm{f}}$ & $432.39^{b}$ & $557.25^{\mathrm{g}}$ & $513.08^{\mathrm{ef}}$ & $2299.81^{\mathrm{f}}$ \\
\hline SEM & & 2.0998 & 3.0600 & 3.2402 & 4.2103 & 4.2356 & 4.1358 & 7.6910 \\
\hline \multicolumn{9}{|c|}{ Effect of Tinospora cordifolia } \\
\hline $0 \%$ & & $118.76^{\mathrm{a}}$ & $201.14^{\mathrm{a}}$ & $356.63^{\mathrm{a}}$ & $407.78^{\mathrm{a}}$ & $457.32^{\mathrm{a}}$ & $440.19^{a}$ & $1981.82^{\mathrm{a}}$ \\
\hline $0.25 \%$ & & $124.29^{\mathrm{bc}}$ & $239.75^{\mathrm{d}}$ & $383.47^{\mathrm{c}}$ & $457.47^{\mathrm{d}}$ & $514.40^{\mathrm{d}}$ & $500.96^{\mathrm{c}}$ & $2220.35^{\mathrm{e}}$ \\
\hline $0.50 \%$ & & $125.54^{\mathrm{bc}}$ & $231.99^{c}$ & $379.36^{\mathrm{bc}}$ & $438.69^{c}$ & $508.60^{\mathrm{cd}}$ & $494.07^{\mathrm{c}}$ & $2178.25^{\mathrm{d}}$ \\
\hline $0.75 \%$ & & $127.76^{\mathrm{c}}$ & $226.86^{c}$ & $376.15^{\mathrm{b}}$ & $435.76^{\mathrm{bc}}$ & $497.11^{b}$ & $483.03^{b}$ & $2146.68^{c}$ \\
\hline
\end{tabular}




\begin{tabular}{llllllll}
$1 \%$ & $122.44^{\mathrm{ab}}$ & $212.10^{\mathrm{b}}$ & $379.55^{\mathrm{bc}}$ & $427.93^{\mathrm{b}}$ & $502.21^{\mathrm{bc}}$ & $475.61^{\mathrm{b}}$ & $\begin{array}{l}2119.85^{\mathrm{b}} \\
5.4384\end{array}$ \\
\hline SEM & 1.4848 & 2.1638 & 2.2912 & 2.9771 & 2.9950 & 2.9244 & \\
\hline Effect of Ascorbic acid & & & & & & & \\
\hline $0 \%$ & 123.75 & $198.68^{\mathrm{a}}$ & $320.73^{\mathrm{a}}$ & $418.96^{\mathrm{a}}$ & $446.34^{\mathrm{a}}$ & $457.22^{\mathrm{a}}$ & $1965.68^{\mathrm{a}}$ \\
$0.025 \%$ & 123.77 & $246.05^{\mathrm{b}}$ & $429.33^{\mathrm{b}}$ & $448.10^{\mathrm{b}}$ & $545.52^{\mathrm{b}}$ & $500.32^{\mathrm{b}}$ & $2293.09^{\mathrm{b}}$ \\
\hline SEM & 0.9391 & 1.3685 & 1.4491 & 1.8829 & 1.8942 & 1.8496 & 3.4395 \\
\hline
\end{tabular}

Table 6: Effect of supplementation of Tinospora cordifolia and ascorbic acid on feed conversion ratio at different weeks

\begin{tabular}{|c|c|c|c|c|c|c|c|}
\hline \multirow{2}{*}{$\begin{array}{l}\text { Treatment } \\
\text { groups }\end{array}$} & \multicolumn{7}{|c|}{ Period (weeks) } \\
\hline & 0-I & I-II & II-III & III-IV & IV-V & V-VI & I-VI \\
\hline \multicolumn{8}{|c|}{ Tinospora cordifolia $\mathrm{X}$ Ascorbic acid } \\
\hline $\mathrm{T}_{1}$ & $1.37^{\mathrm{d}}$ & 1.66 & 1.80 & 1.79 & $2.09^{d}$ & $2.21^{\mathrm{ef}}$ & $1.96^{\mathrm{e}}$ \\
\hline $\mathrm{T}_{2}$ & $1.23^{\mathrm{a}}$ & 1.48 & 1.76 & 1.78 & $1.84^{\mathrm{bc}}$ & $2.15^{\mathrm{d}}$ & $1.81^{\mathrm{c}}$ \\
\hline $\mathrm{T}_{3}$ & $1.28^{\mathrm{ab}}$ & 1.52 & 1.78 & 1.77 & $1.85^{\mathrm{bc}}$ & $2.16^{\mathrm{d}}$ & $1.82^{\mathrm{cd}}$ \\
\hline $\mathrm{T}_{4}$ & $1.30^{\mathrm{bc}}$ & 1.52 & 1.80 & 1.76 & $1.90^{\mathrm{c}}$ & $2.18^{\mathrm{e}}$ & $1.84^{\mathrm{d}}$ \\
\hline $\mathrm{T}_{5}$ & $1.36^{\mathrm{cd}}$ & 1.54 & 1.81 & 1.75 & $1.86^{\mathrm{bc}}$ & $2.25^{\mathrm{f}}$ & $1.85^{\mathrm{de}}$ \\
\hline $\mathrm{T}_{6}$ & $1.31^{\mathrm{bcd}}$ & 1.46 & 1.58 & 1.71 & $1.76^{\mathrm{a}}$ & $2.00^{\mathrm{b}}$ & $1.71^{\mathrm{b}}$ \\
\hline $\mathrm{T}_{7}$ & $1.25^{\mathrm{ab}}$ & 1.34 & 1.56 & 1.65 & $1.70^{\mathrm{a}}$ & $1.91^{\mathrm{a}}$ & $1.65^{\mathrm{a}}$ \\
\hline $\mathrm{T}_{8}$ & $1.25^{\mathrm{ab}}$ & 1.40 & 1.58 & 1.67 & $1.72^{\mathrm{a}}$ & $1.98^{\mathrm{b}}$ & $1.68^{\mathrm{b}}$ \\
\hline $\mathrm{T}_{9}$ & $1.23^{\mathrm{a}}$ & 1.34 & 1.61 & 1.70 & $1.66^{\mathrm{a}}$ & $2.06^{\mathrm{c}}$ & $1.69^{\mathrm{b}}$ \\
\hline $\mathrm{T}_{10}$ & $1.24^{\mathrm{a}}$ & 1.34 & 1.65 & 1.70 & $1.69^{\mathrm{a}}$ & $2.07^{\mathrm{c}}$ & $1.71^{\mathrm{b}}$ \\
\hline SEM & 0.0056 & 0.0071 & 0.0073 & 0.0061 & 0.0089 & 0.0042 & 0.0027 \\
\hline \multicolumn{8}{|c|}{ Effect of Tinospora cordifolia } \\
\hline $0 \%$ & $1.34^{\mathrm{c}}$ & $1.56^{\mathrm{b}}$ & 1.69 & 1.75 & $1.91^{\mathrm{b}}$ & $2.11^{\mathrm{c}}$ & $1.81^{\mathrm{d}}$ \\
\hline $0.25 \%$ & $1.24^{\mathrm{a}}$ & $1.41^{\mathrm{a}}$ & 1.66 & 1.71 & $1.77^{\mathrm{a}}$ & $2.03^{\mathrm{a}}$ & $1.73^{\mathrm{a}}$ \\
\hline $0.50 \%$ & $1.26^{\mathrm{ab}}$ & $1.46^{\mathrm{a}}$ & 1.68 & 1.72 & $1.79^{\mathrm{a}}$ & $2.07^{\mathrm{b}}$ & $1.75^{\mathrm{b}}$ \\
\hline $0.75 \%$ & $1.27^{\mathrm{ab}}$ & $1.43^{\mathrm{a}}$ & 1.70 & 1.73 & $1.78^{\mathrm{a}}$ & $2.12^{\mathrm{c}}$ & $1.76^{\mathrm{bc}}$ \\
\hline $1 \%$ & $1.30^{\mathrm{b}}$ & $1.44^{\mathrm{a}}$ & 1.73 & 1.73 & $1.77^{\mathrm{a}}$ & $2.16^{\mathrm{d}}$ & $1.78^{\mathrm{c}}$ \\
\hline SEM & 0.0136 & 0.0175 & 0.0178 & 0.0148 & 0.0217 & 0.0104 & 0.0066 \\
\hline \multicolumn{8}{|c|}{ Effect of Ascorbic acid } \\
\hline $0 \%$ & $1.31^{\mathrm{b}}$ & $1.55^{\mathrm{b}}$ & $1.79^{\mathrm{b}}$ & $1.77^{\mathrm{b}}$ & $1.91^{\mathrm{b}}$ & $2.19^{\mathrm{b}}$ & $1.85^{\mathrm{b}}$ \\
\hline $0.025 \%$ & $1.26^{\mathrm{a}}$ & $1.38^{\mathrm{a}}$ & $1.60^{\mathrm{a}}$ & $1.69^{\mathrm{a}}$ & $1.70^{\mathrm{a}}$ & $2.00^{\mathrm{a}}$ & $1.69^{\mathrm{a}}$ \\
\hline SEM & 0.0086 & 0.0110 & 0.0112 & 0.0094 & 0.0137 & 0.0065 & 0.0041 \\
\hline
\end{tabular}

to presence of various bioactive compounds as reported earlier in phytochemical analysis of Tinospora cordifolia stem powder (Table 1), which may stimulate the digestion system in poultry and improve the function of liver and increase the pancreatic digestive enzymes. Increased feed intake in Tinospora cordifolia supplemented groups also gain support with the findings of Kamel (2001) who reported that herbs, spices and various plant extracts have appetite and digestion stimulating properties, and antimicrobial effects. In contrast with the findings of
Joshi et al. (2015) who reported that supplementation of Tinospora cordifolia stem powder had no significant effect on overall feed intake. The result in context of ascorbic acid supplementation was in accordance with findings of Elkheir et al. (2008) who reported significant increase in feed intake when broilers were supplemented with vitamin C @ 0.025\%. Further, Elagib and Omer (2012) reported significant increase in feed intake on supplementation of ascorbic acid@350 ppm. The average temperature during experiment was recorded $31^{\circ} \mathrm{C}$, which was higher 
than the painting temperature of $29.4^{\circ} \mathrm{C}$ (Nesheim et $a l ., 1988)$ and well beyond the comfort zone of 12.8 to $23.8^{\circ} \mathrm{C}$ for birds. The adaptation to new challenge of high temperature requires redistribution of body reserves of energy and protein at the cost of decreased body weight that may be one of the reasons for the lowest body weight gain of broilers in control $\left(\mathrm{T}_{1}\right)$ group. Improvement in the body weight gain in Tinospora cordifolia supplementing groups in the present study may be due to improved metabolic efficiency as a result of hepatoprotective activity of Tinospora cordifolia (Bishavi et al., 2002) and also due to the anabolic effect of active principles of herb. Hepatoprotective activity of Tinospora cordifolia in the present study could be explained by the presence of á-Amyrin in extract of Tinospora cordifolia while anabolic property could be due to presence of phytosterol namely campesterol, stigma-sterol and ç-sitosterol in extracts of Tinospora cordifolia. Improvement in the body weight gain in ascorbic acid supplementing groups in the present study are in agreement with the findings of Mckee et al. (1997) who reported that ascorbic acid supplementation alleviates the effect of heat stress on the performance of broiler chickens under heat stress conditions and its antioxidant property, which initiates protein synthesis by bird's enzymatic system (Osawa et al., 1995). Highly significant effect of supplementation of both Tinospora cordifolia and ascorbic acid in various combinations on body weight gain in broiler chicks found in present study could be explained due to antioxidant property of both Tinospora cordifolia and ascorbic acid. The present observation are in accordance with findings of Singh et al. (2009) who reported significant improvement in feed efficiency ratio when broilers were supplemented with Tinospora cordifolia. Improved feed conversion ratio might be due to increased appetite, stomachic, demulcent and tonic activity in addition to immunostimulant, adaptogenic, anabolic and rejuvenative functions of the herbs in the body (Narahari, 1995 and Prajapati, 1997). Flavouring agent, immunostimulant, anabolic and antioxidant activity of Tinospora cordifolia in the present study could be explained by the presence of vanillin lactoside, hexadecanoic acid, octadecanoic acid, campesterol, stigmasterol and ç-sitosterol in extract of Tinospora cordifolia stem powder. The obtained results of study on inclusion of ascorbic acid are in line with the findings of Vathana et al. (2002) who reported significant improvement in FCR on supplementation of ascorbic acid. The improvement in FCR in ascorbic acid supplemented group might be due to antistress effect of ascorbic acid because micronutrient consumption is insufficient to support optimum performance during heat stress as reported by Ferket and Qureshi (1992). On the other hand, ascorbic acid supplementation increases the corticosterone secretion (Bains, 1996) which may be a useful stress management strategy. Highly significant $(\mathrm{p}<0.01$ ) effect of interaction (Tinospora cordifolia $\mathrm{x}$ Ascorbic acid) found in present study are well supported from the findings of Biswas et al. (2012) who reported significant improvement in FCR on inclusion of herb and ascorbic acid in combination. Improved FCR in various combination groups in present study may be due to improvement in nutrient utilization by the birds, which can be explained because of the stimulatory impact of Tinospora cordifolia and ascorbic acid on the GIT and hepatic tissues.

\section{CONCLUSION}

From the present study, it was concluded that supplementation of Tinospora cordifolia at $0.25 \%$ level with ascorbic acid@0.025\% is quite effective and could be viable proposition for lucrative broiler farming for meat production.

\section{ACKNOWLDGEMENTS}

We gratefully acknowledge the help offered by Dean, College of Veterinary and Animal Science, Bikaner and I/C, Poultry Farm, College of Veterinary and Animal Science, Bikaner for providing infrastructure and necessary facilities to conduct the research. We also gratefully acknowledge the help offered by Thermo scientific laboratory for providing GCMS analysis.

\section{REFERENCES}

AOAC. 2005. Official Methods of Analysis, $18^{\text {th }}$ ed. Association of Official Analytical Chemists, Washington, DC, USA.

Bains, B.S. 1996. The role of Vitamin-C in stress management. World Poult., 12(4): 38-41.

Bhushan, S.S., Ramnivas, Sigh, D.P. and Bisen, B. 2013. Impact of herbal based diets on production efficiency of broiler. The Bioscan, 8(1): 119-22. 
Bishavi, B., Roychowdhury, S., Ghosh, S. and Sengupta, M. 2002. Hepatoprotective and immunomodulatory properties of Tinospora cordifolia in $\mathrm{CCl}_{4}$ intoxicated mature albino rats. J. Toxicol. Sci., 27(3): 139-146.

Biswas, P., Sharma, R. K. and Biswas, A. 2012. Effect of dietary supplementation of Ashwagandha (Withania somnifera) and ascorbic acid on production performance of broiler birds. Indian J. Poult. Sci., 47(3): 403-405.

Dhore, R.N., Tangade, S.U. and Dhok, A.P. 2014. Effect of herbal and synthetic vitamin $\mathrm{C}$ supplementation on performance of broilers under intense summer conditions. Indian J. Poult. Sci., 49(1): 48-51.

Donkoh, A. 1989. Ambient temperature: A factor affecting performance and physiological response of broiler chicken. Int. J. Biometerol., 33: 259-265.

Elagib, H.A.A. and Omer, H.M. 2012. Effect of dietary ascorbic acid on performance and immune response of heat stressed broiler chicks. Pak. J. Nutr., 11(3): 216-220.

Elkheir, S., Mohammed, M.K., Ahmed, M.M. and Abdelgadir, S.M. 2008. Effect of feed restriction and ascorbic acid supplementation on performance of broiler chicks reared under heat stress. Res. J. Anim. Vet. Sci., 3: 1-8.

Ferket, P.R. and Qureshi, M.A. 1992. Performance and immunity of heat stressed broilers fed vitamin and electrolyte supplemented drinking water. Poult. Sci., 71(1): 88-97.

Guilhelmelli, F., Vilela, N., Albuquerque, P., Derengowski, L. da S., Silva-pereirai, I. and Kyaw, C. M. 2013. Antibiotic development challenges: the various mechanisms of action of antimicrobial peptides and of bacterial resistance. Front. Microbiol., 4: 353.

Joshi, S.S., Ingle, P.B., Bhagwat, S.R., Pawar, M.M., Prajapati, K.B. and Kulkarni, R.C. 2015. Effect of dietary addition of ashwagandha (Withania somnifera) and guduchi (Tinospora cordifolia) powder on broiler performance. Indian J. Anim. Sci., 85(12): 1358-1361.

Kamel, C. 2001. Tracing methods of action and roles of plant extracts in non-ruminants. In: Recent Advances in Animal Nutrition (eds.). Garns Worthy, P.C. and J. Wiseman, Nottingham, University Press, Nothingham, UK.

Karaman, S., Tarhan, S. and Ergunes, G. 2007. Analysis of indure clamatic data to assess the heat stress for laying hens. Int. J. Nat. Eng. Sci., 1: 65-68.

Kibler, H.H. 1964. Environmental physiology and shelter engineering. LXVII. Thermal effects of various temperaturehumidity combinations on Holstein cattle as measured by eight physiological responses. Research Bulletin of the Missouri Agricultural Experiment Station, pp. 862.
Kramer, C.Y. 1956. Extension of multiple range tests to group means with unequal numbers of replications. Biometrics, 12(3): 307-310.

Kulkarni, R.C., Mandal, A.B., Munj, C.P., Dan, A., Saxena, A. and Tyagi, P.K. 2011. Response of coloured broilers to dietary addition of Tinospora cordifolia during extreme summer. Indian J. Poult. Sci., 46(1): 70-74.

Mckee, J.S., Harrison, P.C. and Riskowski, G.L. 1997. Effect of supplemental ascorbic acid on the energy conversion of broiler chicks during acute heat stress and feed with drawl. Poult. Sci., 76: 1278-1286.

Narahari, D. 1995. Performance promoting ability of livfit in broilers, Poultry Guide, 32: 13-14.

Nesheim, M.C., Austic, R.E. and Card, L.E. 1988. Poultry Production, $12^{\text {th }}$ ed. Lea and Febiger. Philadelphia, USA.

Osawa, T., Sugiyama, Y., Inayoshi, M. and Kawakishi, S. 1995. Antioxidative activity of tetrahydrocurcuminoids. Biosci. Biotechnol. Biochem., 59(9): 1609-1612.

Prajapati, K.S. 1997. Effect of dietary supplementation of LivfitVet premix on performance of broilers. Indian J. Poult. Sci., 32(1): 86-88.

Puvaca, N., Stanaćev, V., Glamocic, D., Levic, J., Peric, L., Stanacev, V. and Milic, D. 2013. Beneficial effects of phytoadditives in broiler nutrition. World's Poult. Sci. J., 69: 27-34.

Saeed, M., Yatao, X., Hassan, F.U., Arain, M.A., Abd ElHack, M.E., Noreldin, A.E. and Sun, C. 2018. Influence of graded levels of 1-theanine dietary supplementation on growth performance, carcass traits, meat quality, organs histomorphometry, blood chemistry and immune response of broiler chickens. Int. J. Mol. Sci., 19: 462.

Singh, V.K., Chauhan, S.S., Ravikanth, K., Maini, S. and Rekhe, D.S. 2009. Effect of dietary supplementation of polyherbal liver stimulant on growth performance and nutrient utilization in broiler chicken. Vet. World, 2(9): 350-352.

Snedecor, G. W. and Cochran, W. G. 2004. Statistical Methods, $8^{\text {th }}$ ed. Oxford and IBH Publishing Company, Kolkata, India.

Vathana, S., Kang, K., Loan, C.P., Thinggaard, G., Kabasa, J.D. and TerMeulen, U. 2002. Effect of vitamin C supplementation on performance of broiler chickens in Cambodia. Conference on International Agricultural Research for Development, Deutscher Tropentag, Witzenhausen, October 9-11, 2002. 